Wittmann, Eveline; Weyland, Ulrike; Nauerth, Annette; Döring, Ottmar; Rechenbach, Simone; Simon, Julia; Worofka, lberé

\title{
Kompetenzerfassung in der Pflege älterer Menschen - Theoretische und domänenspezifische Anforderungen der Aufgabenmodellierung
}

Seifried, Jürgen [Hrsg.]; Faßhauer, Uwe [Hrsg.]; Seeber, Susan [Hrsg.]: Jahrbuch der berufs- und wirtschaftspädagogischen Forschung 2014. Opladen [u.a.] : Verlag Barbara Budrich 2014, S. 53-66. (Schriftenreihe der Sektion Berufs- und Wirtschaftspädagogik der Deutschen Gesellschaft für Erziehungswissenschaft (DGfE); 217)

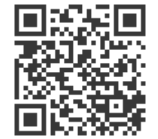

Quellenangabe/ Reference:

Wittmann, Eveline; Weyland, Ulrike; Nauerth, Annette; Döring, Ottmar; Rechenbach, Simone; Simon, Julia; Worofka, Iberé: Kompetenzerfassung in der Pflege älterer Menschen - Theoretische und domänenspezifische Anforderungen der Aufgabenmodellierung - In: Seifried, Jürgen [Hrsg.]; Faßhauer, Uwe [Hrsg.]; Seeber, Susan [Hrsg.]: Jahrbuch der berufs- und wirtschaftspädagogischen Forschung 2014. Opladen [u.a.] : Verlag Barbara Budrich 2014, S. 53-66 - URN:

urn:nbn:de:0111-pedocs-97258 - DOI: 10.25656/01:9725

https://nbn-resolving.org/urn:nbn:de:0111-pedocs-97258

https://doi.org/10.25656/01:9725

in Kooperation mit / in cooperation with:

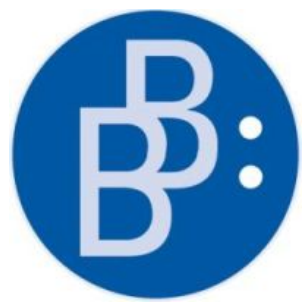

https://www.budrich.de

\section{Nutzungsbedingungen}

Dieses Dokument steht unter folgender Creative Commons-Lizenz: $\mathrm{http}: / /$ creativecommons.org/licenses/by-nc-nd/3.0/de/deed - Sie dürfen das Werk bzw. den Inhalt unter folgenden Bedingungen vervielfältigen, verbreiten und öffentlich zugänglich machen: Sie müssen den Namen des Autors/Rechteinhabers in der von ihm festgelegten Weise nennen. Dieses Werk bzw. dieser Inhalt darf nicht für kommerzielle Zwecke verwendet werden und es darf nicht bearbeitet, abgewandelt oder in anderer Weise verändert werden.

Mit der Verwendung dieses Dokuments erkennen Sie die Nutzungsbedingungen an.

\section{Terms of use}

This document is published under following Creative Commons-License: http://creativecommons.org/licenses/by-nc-nd/3.0/de/deed.en - You may copy, distribute and transmit, adapt or exhibit the work in the public as long as you attribute the work in the manner specified by the author or licensor. You are not allowed to make commercial use of the work or its contents. You are not allowed to alter, transform, or change this work in any other way.

By using this particular document, you accept the above-stated conditions of use.

\section{Kontakt / Contact:}

\section{peDOCs}

DIPF | Leibniz-Institut für Bildungsforschung und Bildungsinformation Informationszentrum (IZ) Bildung

E-Mail: pedocs@dipf.de

Internet: www.pedocs.de

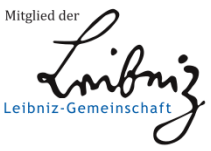




\section{Jahrbuch der berufs- und wirtschaftspädagogischen Forschung 2014}

Jürgen Seifried, Uwe Faßhauer Susan Seeber (Hrsg.)

DGE Deutsche Gesellschaft 
Schriftenreihe der Sektion

Berufs- und Wirtschaftspädagogik der Deutschen Gesellschaft für Erziehungswissenschaft (DGfE) 
Jürgen Seifried

Uwe Faßhauer

Susan Seeber (Hrsg.)

\section{Jahrbuch der berufs- und wirtschaftspädagogischen Forschung 2014}

Verlag Barbara Budrich

Opladen • Berlin • Toronto 2014 
Bibliografische Information der Deutschen Nationalbibliothek

Die Deutsche Nationalbibliothek verzeichnet diese Publikation in der Deutschen Nationalbibliografie; detaillierte bibliografische Daten sind im Internet über http://dnb.d-nb.de abrufbar.

(C) Dieses Werk ist im Verlag Barbara Budrich erschienen und steht unter folgender Creative Commons Lizenz: http://creativecommons.org/licenses/by-nc-nd/3.0/de/ Verbreitung, Speicherung und Vervielfältigung erlaubt, kommerzielle Nutzung und Veränderung nur mit Genehmigung des Verlags Barbara Budrich.

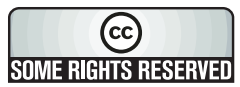

Dieses Buch steht im OpenAccess Bereich der Verlagsseite zum kostenlosen Download bereit (http://dx.doi.org/10.3224/84740164)

Eine kostenpflichtige Druckversion (Printing on Demand) kann über den Verlag bezogen werden. Die Seitenzahlen in der Druck- und Onlineversion sind identisch.

$$
\begin{array}{ll}
\text { ISBN } & 978-3-8474-0164-3 \text { (Paperback) } \\
\text { eISBN } & \mathbf{9 7 8 - 3 - 8 4 7 4 - 0 4 4 1 - 5 ~ ( e B o o k ) ~} \\
\text { DOI } & 10.3224 / 84740164
\end{array}
$$

Umschlaggestaltung: bettina lehfeldt graphic design, Kleinmachnow

Typografisches Lektorat: Judith Henning, Hamburg

Verlag Barbara Budrich, http://www.budrich-verlag.de 


\section{Inhaltsverzeichnis}

Vorwort 9

\section{Teil I: Perspektiven der historischen Berufsbildungsforschung}

Frank-Lothar Kroll

Möglichkeiten und Notwendigkeiten historiographischen Arbeitens in der Berufs- und Wirtschaftspädagogik........................................................ 1

Volker Bank, Annekathrin Lehmann

Theodor Franke. Sächsischer Pionier wirtschaftspädagogischen

Denkens in Deutschland

Marcel Schweder

Lehrerarbeit im Strafvollzug - Ein Diskurs aus historischer Sicht

\section{Teil II: Kompetenzmodellierung, -messung und -förderung}

Eveline Wittmann, Ulrike Weyland, Annette Nauerth, Ottmar Döring, Simone Rechenbach, Julia Simon, Iberé Worofka Kompetenzerfassung in der Pflege älterer Menschen - Theoretische und domänenspezifische Anforderungen der Aufgabenmodellierung 53

Simon Heinen, Martin Frenz, Christopher M. Schlick

Bildung für nachhaltige Entwicklung in der Gebäudeenergieberatung - Entwicklung eines Kompetenzmodells für die Förderung reflexiver Handlungsfähigkeit

Diana Stuckatz, Cornelia Wagner

Qualifizierungsangebote in der Pflegehilfe für Personen mit geringen Grundbildungskenntnissen - Empirische Studien zur Entwicklung von Lehr-Lern-Umgebungen und Arbeitsmaterialien. 81 


\section{Teil III: Gestaltung und Analyse von Lehr-Lern-Prozessen}

Eva Höpfer, Andrea Reichmuth, Doreen Holtsch, Franz Eberle

Wer sieht was? - Zum Umgang mit unterschiedlichen Sichtweisen auf

Unterricht am Beispiel des kaufmännischen Berufsschulunterrichts 95

Mandy Hommel

Sozial geteilte Reflexion - eine explorative Studie im

Mathematikunterricht. 109

Gerhard Minnameier, Rico Hermkes

„Kognitive Aktivierung“ und „konstruktive Unterstützung“ als Lehr-

Lern-Prozess-Größen - Eine Konzeption im rechnungswesen-

didaktischen Kontext

\section{Teil IV: Lehrerbildung und pädagogische Professionalität}

Nicole Kimmelmann, Johannes Lang

Lehramtsstudierende mit Migrationshintergrund und ihre

Schwierigkeiten an der Universität

Robert W. Jahn

Stützlehrer als neuer pädagogischer Profi in der Beruflichen Bildung?! .... 147

Sabrina Berg

Pädagogische Praxis und Reproduktion sozialer Ungleichheit - zur

Berücksichtigung sozialer Herkunft im Wirtschaftsunterricht..... 161

\section{Teil V: Perspektiven der Berufsbildungsforschung}

\section{Miriam Voigt}

Neo-institutionalistische und mikropolitische Prozesse in

Schulentwicklungsprojekten

Lara Forsblom, Lucio Negrini, Jean-Luc Gurtner \& Stephan Schumann

Lehrvertragsauflösungen und die Rolle der betrieblichen Auswahl von

Auszubildenden 
Marius R. Busemeyer

Organisierte Interessen, Parteipolitik und institutioneller Wandel im deutschen Berufsbildungssystem

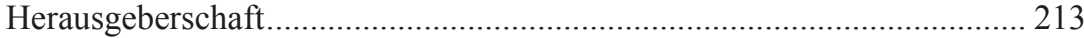

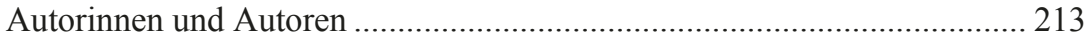




\title{
Kompetenzerfassung in der Pflege älterer Menschen - Theoretische und domänenspezifische Anforderungen der Aufgabenmodellierung ${ }^{1}$
}

\author{
Eveline Wittmann, Ulrike Weyland, Annette Nauerth, Ottmar Döring, \\ Simone Rechenbach, Julia Simon, Iberé Worofka
}

\section{Einleitung}

Das im Rahmen der ASCOT-Initiative des BMBF zur „Technologieorientierten Kompetenzmessung in der Berufsbildung“ durchgeführte Verbundprojekt ${ }^{2}$ „Entwicklung und Erprobung von technologieorientierten Messinstrumenten zur Feststellung der beruflichen Handlungskompetenz in der Pflege älterer Menschen (TEMA)“ zielt auf die Konstruktion eines technologiebasierten Erhebungsinstruments für diesen Bereich ab. Hiermit soll eine Grundlage für eine standardisierte, repräsentative Testung beruflicher Handlungskompetenz zum Ende der Berufsausbildung geschaffen werden. Das Testinstrument wird in einer Validierungsstudie im Altenpflegeberuf einer empirischen Prüfung unterzogen. Die Chancen solcher technologieorientierten Testkonstruktionen liegen gegenüber Paper- und Pencil-Tests unter anderem in einer erhöhten Authentizität, insofern als diese eine verbesserte Situationsdarstellung und eine kontextualisierte Aufgabendarstellung ermöglichen.

Für Pflegeberufe ist dabei die Besonderheit zu beachten, dass hier die unmittelbare Arbeit mit Menschen im Vordergrund steht. Reale berufliche Handlungskontexte und Handlungen in eine technologieorientierte Abbildung zu übersetzen, erfordert daher größere Transformationsleistungen als in anderen Berufen. Insbesondere ist eine Simulation von Handlungszusammenhängen und -abläufen, wie dies in technologieaffineren Ausbildungsberufen geschieht (z. B. Geißel/Hedrich 2011, S. 14, Gschwendtner/Geißel/Nickolaus 2010, S. 263), aufgrund der interaktiven Struktur des Pflegehandelns proble-

1 Mit dem Begriff „Modellierung“ ist hier eine aus Teilmodellierungen, u. a. zu Kompetenzen und Aufgaben, aber auch zur Messung und zur technologischen Umsetzung bestehende heuristische Modellierung gemeint. Sie liegt der Instrumentierung zugrunde und erlaubt es, Überlegungen, auf denen die Instrumentenerstellung basiert, sowie deren Gültigkeit nachvollziehbar zu machen und nach Möglichkeit im Rahmen des vorliegenden Projektes oder weiterer Projekte auch empirisch zu prüfen (z. B. Mislevy/Steinberg/Almond 1999, S. 6).

2 Verbundpartner sind das Forschungsinstitut Betriebliche Bildung (f-bb), die Otto-FriedrichUniversität Bamberg, die Fachhochschule Bielefeld und das Deutsche Institut für Internationale Pädagogische Forschung (DIPF). 
matisch und, wenn überhaupt, nur mit einem großen Aufwand realisierbar. Um die für die Abbildung von Handlungskompetenz erforderliche Handlungsorientierung zu erzeugen, werden im Projekt TEMA daher komplexe und realitätsnah abgebildete kontextuelle Stimuli erzeugt, welche eine Vielzahl unmittelbarer Handlungsaufforderungen enthalten. Die Stimuli werden videografisch dargestellt.

Der Erstellung solcher komplexen, unter der Perspektive von Authentizität konstruierter Situationen und Testaufgaben liegt allerdings notwendigerweise eine Reihe von Transformationsschritten und damit von Strukturierungen, aber möglicherweise auch Verkürzungen realer betrieblicher Handlungsanforderungen zugrunde. Diese Transformation kann implizit oder modellgeleitet expliziert erfolgen. Im Falle einer Explizierung kann hierbei von einer systematischen Inszenierung von Authentizität gesprochen werden. Im Sinne einer Modellierung von Achtenhagen und Winther (2009, S. 11, Abb. 1) läge ein erster Transformationsschritt in der wissenschaftlichen, empirisch fundierten Modellierung der Realität, hier von kontextuellen und situativen Erfordernissen des Pflegehandelns, auf die eine fachdidaktisch begründete Aufgabenmodellierung aufbauen würde. Letzterer würden nach Möglichkeit authentische Situationen aus Situationsbeschreibungen von Pflegepersonal zugrunde legen (vgl. auch Wittmann 2003). Das Verfahren wäre also sinnvollerweise rekursiv.

\section{Abb. 1: Modellierungsschritte bei der Inszenierung von Authentizität}

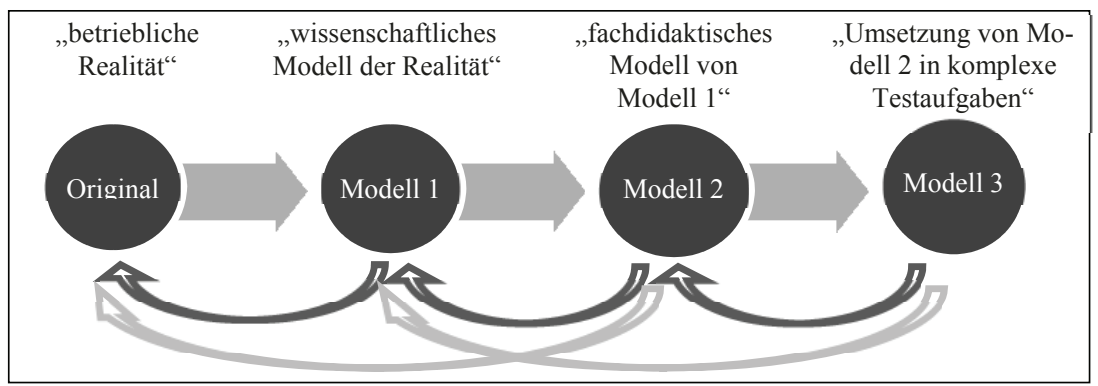

Quelle: Achtenhagen \& Winther 2009, S. 11

In Anbetracht des verfügbaren Rahmens wird die Darstellung auf den Aspekt der fachdidaktischen Aufbereitung und Modellierung (Modell 2 der Abb. 1) gelegt. Die Fragestellung, die im Folgenden thematisiert wird, lautet: Welche Modellierungen liegen diesbezüglich im Projekt TEMA der Inszenierung von Situationen und Handlungsaufforderungen zugrunde? Der Beitrag ist damit im Kern auf eine berufs- und wirtschaftspädagogische bzw. fachdidaktische Begründung für das im Projekt gewählte Vorgehen fokussiert. 


\section{Ausgangslage: Domänenspezifisches Kompetenzmodell und konzeptionelle Grundlagen der Aufgabenmodellierung}

Kompetenzen können verstanden werden ,als kontextspezifische [...] Leistungsdispositionen, die sich funktional auf Situationen und Anforderungen in bestimmten Domänen beziehen“ (Klieme/Leutner 2006, S. 879; Hervorh. d. Verf.). Im Rahmen der ASCOT-Initiative geht es dabei um die Erhebung berufsfachlicher Kompetenzen (BMBF 2011). Im Sinne von Pätzold (2006, S. 175) sind die zentralen Komponenten von Fachkompetenz zu sehen in „einem fachgerechten Urteil und [...] einem verantwortlichen, an Normen und Vorschriften des beruflich organisierten Arbeitens ausgerichteten Handeln [...] - verstanden als zielgerichtete, bewusste, zwischen Alternativen entscheidende, Mittel auswählende, Nebenwirkungen abschätzende, kritisch-reflektierende Tätigkeit.“ Im Projekt TEMA wird dabei ein domänenspezifischer Zuschnitt von Fachkompetenz vorgenommen: Fachdidaktische Konzeptionen definieren „Sozialkompetenz zugleich als Bestandteil von Fachkompetenz" (Friese 2010, S. 326). Dieser Zuschnitt wird mit dem im Kern interaktiven Charakter pflegerischer Diagnostik und Therapie einerseits und der berufsfachlich bedingten Erfordernisse, Einschränkungen und Zuschnitte des interaktiven pflegerischen Handelns andererseits begründet (vgl. ausführlich Döring et al. 2011, S. 53ff.).

Abb. 2: Modellierung der berufsfachlichen Kompetenz

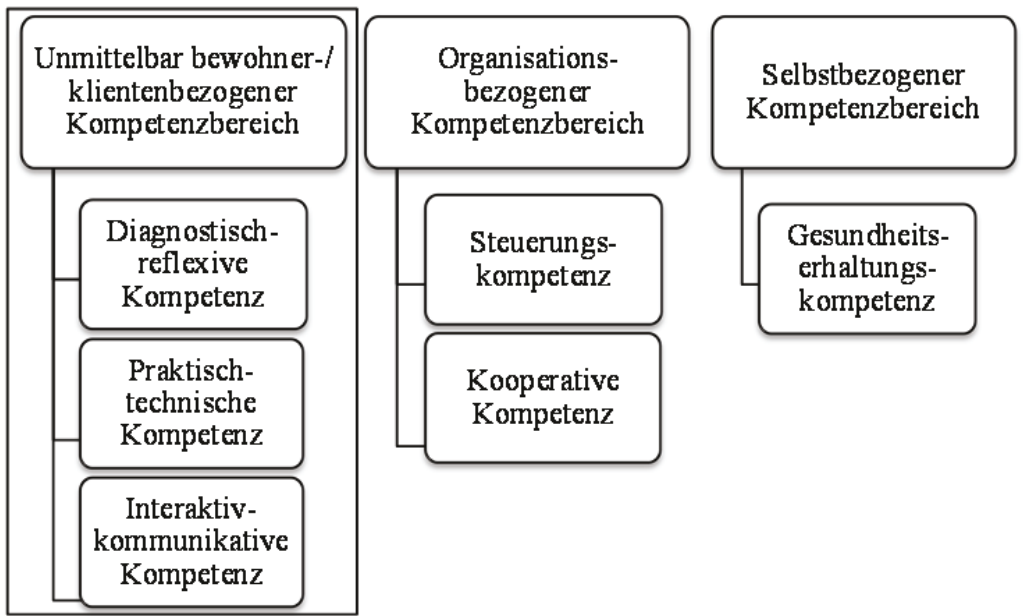

Quelle: eigene Darstellung 
Das domänenspezifische Kompetenzmodell (Abb. 2) differenziert sechs Teilkompetenzen aus, von denen drei sich unmittelbar auf die zu pflegende Person und deren Angehörige richten, wobei die Operationalisierung im Rahmen des Projekts auf diesen Bereich begrenzt wird. Weitere, nicht operationalisierte Kompetenzbereiche betreffen die Arbeitsorganisation und das selbstbezogene Handeln (vgl. Döring et al. 2011, S. 53ff.). Die folgenden Überlegungen zur Inszenierung von Authentizität der Situationen und Handlungsaufforderungen im entwickelten Testinstrument werden exemplarisch anhand der ,praktisch-technischen Teilkompetenz“ als Komponente des unmittelbar patientenbezogenen Kompetenzbereichs verdeutlicht. Sie wird in diesem Projekt definiert als die Kompetenz zu Interventionen bezogen auf zu pflegende Personen und ihre unmittelbare Umgebung mittels pflegerischer und medizinischer Techniken, Methoden und Hilfsmittel im Hinblick auf den umfassenden Pflegebedarf. ${ }^{3}$ Letzterer basiert auf der objektiven und subjektiven Pflegebedürftigkeit.

Im Sinne einer validen Assessment-Argumentation sind im Rahmen der Aufgabenmodellierung Überlegungen zur Frage zugrunde zu legen, welches Verhalten bei welchen Aufgaben in welcher Situation erkennen lässt, dass die gemäß Kompetenzmodell zu messende Kompetenz gegeben ist (Mislevy/Almond/Lukas 2004, S. 3f. unter Rückgriff auf Messick 1994, S. 17) - hier also die praktisch-technische Kompetenz als Teilkompetenz der beruflichen Handlungskompetenz in der Pflege älterer Menschen, weiter konkretisiert um das angenommene Kompetenzniveau. Solche Überlegungen dienen dazu, die Aufgabenentwicklung und deren Systematik sowie das auf dieser Basis erstellte Erhebungsinstrument zu begründen, aber auch einer wissenschaftlichen Kritik zugänglich zu machen. Eine „Aufgabe“ verstehen Mislevy, Steinberg und Almond (1999) mit Haertel und Wiley (1993, S. 361) als ,goal-directed human activity to be pursued in a specified manner, context, or circumstance." In diesem Sinne sollte eine Aufgabenmodellierung den Rahmen für Situationen beinhalten, in denen Probanden handeln, d. h. unter anderem

- die Modellierung des Stimulusmaterials, der handlungsauffordernden Merkmale und der Umgebungen, in denen Probanden tätig werden (Aufgabenpräsentation), sowie

- Überlegungen zum Aufgabendesign und zur Schwierigkeitszuordnung (Aufgabenmodellvariablen) (Mislevy/Steinberg/Almond 1999, S. 18, Mislevy/Almond/Lukas 2004, S. 11f., S. 31).

Inhaltliche Basis der Aufgabenmodellierung sollten hierbei nach Auffassung von Mislevy, Steinberg und Almond (1999, S. 24) theoretische und konzeptuelle Fundierungen sein. Zentral ist im Projekt TEMA hierfür vor dem Hintergrund des eingangs skizzierten Zuschnitts bei der Umsetzung von Hand-

3 Von der ,interaktiv-kommunikativen Kompetenz“ wird die praktisch-technische Kompetenz insofern abgegrenzt, als dort die Beziehungsgestaltung im Zentrum steht. 
lungsorientierung das im Folgenden dargestellte, fachdidaktisch begründete Situationskonzept, auf das dementsprechend ausführlich eingegangen wird.

\section{Aufgabenmodellierung}

\subsection{Vorüberlegungen zur Modellierung betrieblicher Situationen}

Der Erstellung des Aufgabenmodells gingen umfangreiche Arbeiten zur Modellierung der betrieblichen Realität (siehe wissenschaftliches Modell der Realität in Abb.1) voraus, auf die im Rahmen dieses Beitrags nicht genauer eingegangen wird. Hierzu gehören Experteninterviews, curriculare Analysen und Expertenvalidierungen (Abb. 3). Besonders zu verweisen ist auf vorliegende, auf qualitativer Empirie basierende Modellierungen aus der Pflegedidaktik zu Merkmalen des Pflegehandelns und dessen Erlernens. So stellen Fichtmüller und Walter (2007, S. 357) für das Erlernen des Pflegehandelns im Kontext des Krankenhauses heraus, dass Mehrfachhandeln im Sinne parallel zu versorgender Patientinnen und Patienten von den Pflege Lernenden als ein problematischer, die Komplexität des zu erlernenden Pflegehandelns erhöhender Aspekt wahrgenommen wird. Darüber hinaus wurde den Überlegungen unter anderem eine am Standort Universität Bamberg außerhalb des TEMA-Verbundprojektes durchgeführte Zusatzstudie zu herausfordernden Situationen in Pflegesettings zugrunde gelegt. Demzufolge stellen Non-Compliance und Aggressivität von zu Pflegenden sowie Zeitdruck wesentliche Merkmale besonders herausfordernder Situationen des Pflegehandelns dar (Wittmann et al., in Vorbereitung).

Die genannten Aspekte sind für die Aufgabenmodellierung insofern besonders bedeutsam, als sie, wie im Weiteren anhand der praktisch-technischen Teilkompetenz exemplarisch verdeutlicht wird, als fachspezifische schwierigkeitskonstituierende Aspekte in die Schwierigkeitsmodellierung eingehen. Damit stellen sie gleichzeitig wesentliche Merkmale der konkreten Ausgestaltung der den Probanden präsentierten Situationen und Handlungsaufforderungen dar. Sie dienen also im Sinne der Erläuterungen zu Abb. 1 der Konkretisierung des im Folgenden präsentierten abstrakten Konzepts zu handlungsauffordernden Merkmalen. 
Abb. 3: Modellierung betrieblicher Realität

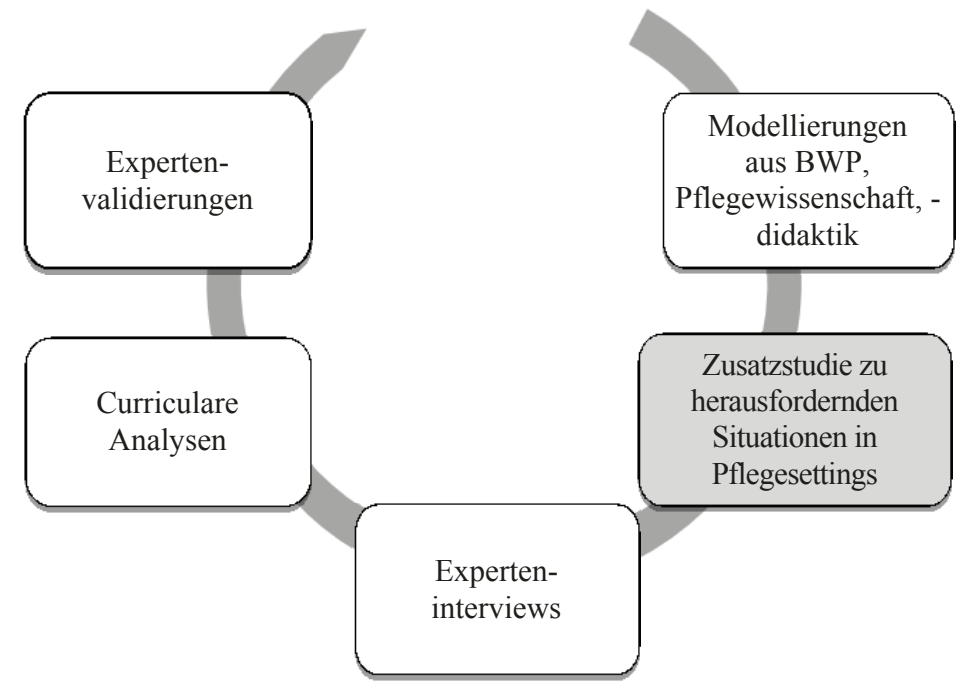

Quelle: eigene Darstellung

\subsection{Situationskonzept}

Wie einleitend beschrieben, stellt das fachdidaktisch begründete Situationskonzept einen Kernaspekt der Aufgabenmodellierung im Projekt TEMA dar. Es liegt den erarbeiteten Aufgaben durchgängig zugrunde. Das Konzept basiert auf einer pflegedidaktischen Modellierung der Pflegesituation, welche Hundenborn und Knigge-Demal (Hundenborn 2007, S. 46) in Orientierung an einer Modellierung der kaufmännischen Fallstudiendidaktik nach Kaiser vornehmen (Abb. 4). Nach Kaiser (1985, S. 35) sind Situationen hierbei „Orte, an denen menschliche Handlungsfähigkeit eingefordert ist, an denen sie sich äußert, an denen sie sich bewährt oder scheitern kann“". Die Generierung der Situationen dient mithin der Erzeugung von Handlungsaufforderungen, von denen angenommen wird, dass sich jeweils die unterschiedlichen Teilkompetenzen des in Abb. 2 dargestellten TEMA-Kompetenzmodells hierin abbilden lassen. Dementsprechend enthält das Situationskonzept Annahmen über verallgemeinerbare Merkmale pflegerischer Anforderungssituationen.

Dem Modell von Hundenborn und Knigge-Demal (Hundenborn 2007, S. 46) zufolge sind Pflegesituationen sowie Handlungen in diesen Situationen regelmäßig dadurch gekennzeichnet, dass sie

- sich auf bestimmte objektive Pflegeanlässe beziehen, die den Pflegebedarf begründen, der sich wiederum sowohl unmittelbar aus Krankheiten, 
Unfällen und Behinderung als auch in besonderen Lebens- und Entwicklungsphasen, z. B. aus altersbedingten Veränderungsprozessen, ergibt;

- die subjektiven Pflegeanlässe bzw. das subjektive Erleben und Verarbeiten in den Pflegesituationen im Hinblick auf subjektive Deutungen, Einschätzung von Ressourcen und Belastungsgrenzen sowie Bewältigungsstrategien integrieren, die je nach Zielperspektive kurativ, rehabilitativ, palliativ, präventiv und gesundheitsfördernd ausgerichtet sind;

- in einem bestimmten institutionellen Kontext stattfinden, z. B. einem Setting der ambulanten Pflege, in welchem Angehörige verstärkt in den Pflegeprozess einzubinden sind, oder einem Setting der stationären Pflege, in welcher etwa die angesprochene Problematik paralleler Handlungsstränge verstärkt auftreten kann;

- durch spezifische Interaktionsstrukturen gekennzeichnet sind, die sich aus den Rollen der Interaktionsteilnehmerinnen und -teilnehmer ergeben, um ein gemeinsames Pflege- bzw. Behandlungsziel mit den zu Pflegenden und deren Bezugspersonen auszuhandeln.

Abb. 4: Fachdidaktische Modellierung einer Pflegesituation

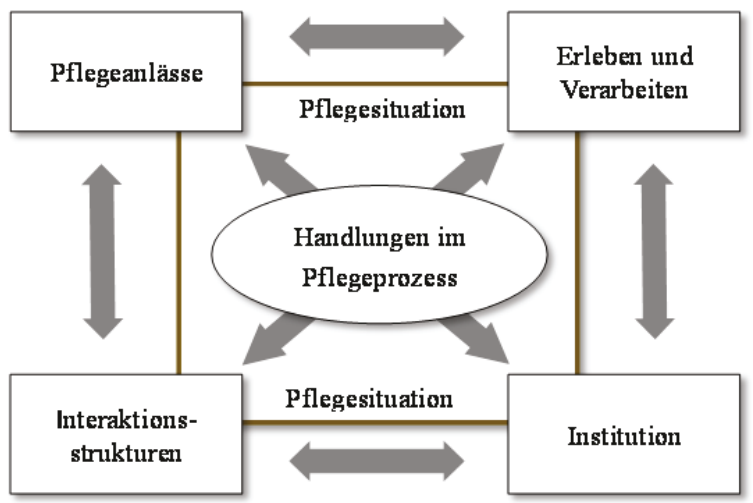

Quelle: Hundenborn/Knigge-Demal (leicht modifiziert nach Hundenborn 2007, S. 46)

Als fünftes konstitutives, formales Element einer Pflegesituation, ist - neben den bisher genannten materialen Komponenten - der sogenannte Pflegeprozess selbst zu benennen. Nach Hundenborn (2007, S. 48) entspricht je nach Pflegeprozessmodell ,das Handeln der Pflegeperson in der Pflegesituation in 
seiner formalen Struktur einer spezifischen Schritt- bzw. Phasenfolge von Einschätzung, Planung, Durchführung und Beurteilung“. ${ }^{4}$

Das Situationskonzept mit den beschriebenen konstitutiven Merkmalen zieht sich durch die im TEMA-Projekt generierten Situationen. Hierbei wurden im Projekt zwölf Situationen für drei typische, durch Experten validierte Settings der Pflege älterer Menschen in der Altenpflege generiert. Auf die konkrete Auswahl kann im Rahmen dieses Beitrags nicht genauer eingegangen werden. Vielmehr wird exemplarisch dargestellt, inwieweit sich auf Basis dieses Situationskonzepts Handlungsaufforderungen erzeugen lassen, mit deren Bewältigung die praktisch-technische Kompetenz als Bestandteil der beruflichen Handlungskompetenz in der Pflege älterer Menschen abgebildet werden kann. Konkret wird anhand eines Beispiels die Umsetzung des Situationskonzepts im Rahmen der Aufgabenpräsentation verdeutlicht sowie hieran anschließend der Frage nachgegangen, welche Überlegung hier der Aufgabengestaltung zur Abbildung der praktisch-technischen Teilkompetenz zugrunde gelegt werden.

\subsection{Aufgabenpräsentation und Variablen des Aufgabenmodells}

Die Umsetzung des Situationskonzepts kann exemplarisch anhand eines Beispiels verdeutlicht werden, dessen Setting eine Wohngruppe für dementiell erkrankte ältere Menschen darstellt. Objektive Pflegeanlässe sind hier zunächst die demenzbedingten, individuell unterschiedlich eingeschränkten funktionellen Lebensaktivitäten, die sich im subjektiven Erleben u. a. in einer eingeschränkten Wahrnehmungsfähigkeit äußern. Aufgrund des institutionellen Settings einer Wohngruppe für dementiell erkrankte ältere Menschen können hier Interaktionssituationen auftreten, in denen mehrere dementiell erkrankte Bewohnerinnen und Bewohner gleichzeitig beteiligt sind. Um ihnen professionell begegnen zu können, müssen Pflegekräfte - unter Berücksichtigung der objektiven und subjektiven Pflegeanlässe - einrichtungsspezifische Konzepte zur Identitätssicherung und geragogische Konzepte in

4 Der WHO-Pflegeprozess ist ein interaktiver Vorgang, in welchem die Pflegeperson mit der zu pflegenden Person und deren Bezugspersonen in einem dynamischen Prozess den Pflegebedarf, die Pflegeziele, die Pflegeinterventionen und die Pflegeergebnisse einschätzt und beurteilt - unterteilt in die vier Phasen: (1) Assessment, (2) Planung, (3) Durchführung und (4) Evaluation und Feedback. Auf diese Art und Weise hilft der Pflegeprozess dem Patienten/Klienten/Bewohner dabei, mehr Verantwortung für die Selbstpflege zu übernehmen, bietet einen Feedback-Mechanismus, um die Qualität der erbrachten Pflege zu verbessern und bietet die Möglichkeit, den Informationsstand über den Pflegebedarf zu erweitern (in Anlehnung an Ashworth et al. 1987, S. 34ff.). Eine gewisse Deckungsgleichheit mit dem Handlungsbegriffs liegt nahe, die Abgrenzung zum Konzept der vollständigen Handlung ist aber nicht trivial. So sind z. B. Anfang und Ende im pflegerischen Handeln nicht immer eindeutig zu bestimmen. Darüber hinaus sind die zu Pflegenden partizipativ in die Phasen des Pflegeprozesses involviert. 
den Interaktions-/Beziehungsprozess integrieren können (z. B. Hundenborn/Brühe 2007). Die videografische Darstellungsweise ermöglicht es, solche komplexen Anforderungskonstellationen der Pflege sowie das Verhalten der Beteiligten anschaulich darzustellen, ohne die Aufgabenstimuli textlich $\mathrm{zu}$,überfrachten“ oder personales Verhalten durch textliche Darstellungen zu verfälschen. ${ }^{5}$ Die grundlegende settingbezogene Konstellation wird den Probandinnen und Probanden dazu jeweils unter Rückgriff auf den erläuterten fachdidaktischen Begründungszusammenhangs systematisch videografisch vorgestellt. Dadurch werden den Probandinnen und Probanden also wesentliche setting- (z. B. Art und Ausstattung des Settings, personale Konstellation, Tagesablauf) und bewohnerbezogene Informationen (wie vorliegende Pflegediagnosen, typische Verhaltensweisen) vorab gegeben, die in der Altenpflege typischerweise vorhanden sind. Auch in den ebenfalls videografisch aufbereiteten aktuellen Situationen sind die fachdidaktischen Konstruktionsüberlegungen maßgeblich. Hier sind jedoch verstärkt situationsbezogene Wahrnehmungs- und Interpretationsleistungen erforderlich. Vor diesem Hintergrund ergibt sich eine komplexe, pflegetypische Konstellationen nachvollziehbar variierende, gleichzeitig aber möglichst realitätsnahe Umgebungs- und Situationsdarstellung. ${ }^{6}$

Aufgaben zur praktisch-technischen Teilkompetenz zielen gemäß der bereits dargestellten Kompetenzdefinition auf Interventionen, die mittels pflegerischer und medizinischer Techniken, Methoden und Hilfsmittel durchgeführt werden und den individuellen Pflegebedarf bearbeiten, wobei nicht nur die unmittelbar zu pflegende Person, sondern auch die Umgebung zu beachten sind - im Falle des beschriebenen Settings etwa andere anwesende Bewohnerinnen und Bewohner. Mit dem ,individuellen Pflegebedarf“ ist in diesem Kontext neben der Bearbeitung von krankheits- und entwicklungsbedingten Pflegebedarfen auch die positive Beeinflussung des emotionalen Zustandes sowie der sozialen Situation der zu Pflegenden gemeint (WHO 1996, Hundenborn 2007, S. 46ff., Wingenfeld/Büscher/Gansweid 2008, BMG 2009).

Eine Problematik der Operationalisierung der praktisch-technischen Kompetenz ist, dass Performanz im Rahmen einer videobasierten Aufgaben-

5 Auf die videografischen Darstellungen, die mit diesem Stimulus möglicherweise verbundenen Validitätsprobleme und den Umgang hiermit soll und kann im Rahmen dieses Beitrags nicht detailliert eingegangen werden. Es kann jedoch festgehalten werden, dass auch hier Experteneinschätzungen der videografischen Situationsdarstellungen eingeholt wurden. Weitere Validierungsschritte werden für erforderlich gehalten.

6 Die Filmaufnahmen fanden in authentischen Pflegeumgebungen mit den jeweiligen Ausstattungen und Hilfsmitteln statt. Die Bewohner wurden durch erfahrene Laienschauspieler/Simulationspatienten dargestellt. Teilweise wurde die videografische Darstellung durch weitere Elemente wie Dokumente bzw. Fotos unterstützt, wo dies für eine möglichst authentische, aber auch Erinnerungseffekte vermeidende Situationsdarstellung sinnvoll und erforderlich erschien. 
darstellung nicht erfasst werden kann. Allerdings wird mit dem Konstrukt „Handlungskompetenz" auf das dem Handeln zugrundeliegende situationsbezogene Vermögen abgehoben, zu planen und abzuschätzen, zu entscheiden und zu reflektieren. Aufgabenformate zur Abbildung der praktisch-technischen Kompetenz beinhalten vor diesem Hintergrund unter anderem

- die Abfrage von in Situationen ,eingetaktetem“ Handlungswissen (Wie vorgehen?),

- Handlungsentscheidungen in situativen Kontexten (Welche Handlung zuerst vornehmen?),

- die Bewertung beobachteter (simulierter) Handlungen (Wie gut wurde eine Handlung durchgeführt?) sowie

- Konzepte zur Begründung von Handlungsplänen, Handlungsentscheidungen und der Bewertung von Handlungen (Weshalb sollte in einer bestimmten Weise gehandelt werden bzw. aus welchen Gründen sollten bestimmte Handlungen unterlassen werden?).

Eine Beispielaufgabe lautet etwa: „In welcher Reihenfolge kümmern Sie sich um die Bewohner, um ihnen in der Situation gerecht zu werden?" Die Lösungen der Aufgaben erfordern hierbei die Einbeziehung spezifischer Interaktionsstrukturen des jeweiligen Settings sowie der Dringlichkeit von Pflegeanlässen und des subjektiven Erlebens. In Betracht zu ziehen sind im Setting der Wohngruppe für Demenzerkrankte z. B. krankheitsbedingte Einschränkungen der Wahrnehmung und der Kommunikation und hieraus resultierendes Verhalten.

Diese Aspekte finden auch in der heuristischen Schwierigkeitsmodellierung des Projekts TEMA Berücksichtigung, in die darüber hinaus die zuvor beschriebene qualitative Empirie eingeht. Pflegepersonen auf einem hohen Kompetenzniveau führen Pflegemaßnahmen dieser Modellierung zufolge unter anderem auch bei mehreren parallel verlaufenden Handlungssträngen und

- in Anbetracht von Störungen durch Pflegeerfordernisse weiterer Pflegepersonen oder

- $\quad$ bei Non-Compliance oder Aggressivität der zu Pflegenden sowie

- unter Zeitdruck

jederzeit unter Berücksichtigung der aktuellen Bedürfnisse und des Befindens der zu Pflegenden sicher durch. Die Schwierigkeitsmodellierung enthält weitere Merkmale, wie die Komplexität der durchzuführenden Einzelhandlungen, betont aber gerade auch pflegespezifische Elemente, insbesondere die Tatsache, dass es hier um die Durchführung von Techniken am Menschen geht. In ihr spiegeln sich alle vier Aspekte des Situationskonzepts wider: Die Modellierung beinhaltet sowohl die Interaktionsstrukturen und deren spezifische Ausprägungen in den Settings - also die Frage, ob eine oder mehrere Personen pflegerisch zu versorgen sind - als auch Pflegeerfordernisse unter 
Berücksichtigung aktueller Bedürfnisse und aktuellen Befindens sowie hieraus ggf. resultierende Non-Compliance und Aggressivität; darüber hinaus wird, soweit im Rahmen der technologischen Instrumentierung abbildbar, der Aspekt des Zeitdrucks einbezogen, welcher sich setting- und pflegeanlassbezogen ergeben kann.

Die Schwierigkeit der auf Basis der vorstehenden Überlegungen erarbeiteten Aufgaben war bereits Gegenstand von Experteneinschätzungen und ersten Feldstudien, die allerdings nur bedingt Rückschlüsse auf tatsächliche Aufgabenschwierigkeiten am Ende der Pflegeausbildung zulassen. Inwieweit und ggf. in welchen Kombinationen die theoretisch vermuteten schwierigkeitsbestimmenden Merkmale bei dieser Personengruppe auch empirisch wirksam werden, ist daher Bestandteil laufender und künftiger Prüfungen.

\section{Fazit}

Die Inszenierung von Authentizität im Rahmen einer technologie-orientierten, videobasierten Simulation stellt in der Pflege eine besondere Herausforderung dar. Diese besteht nicht nur in der mangelnden Simulierbarkeit von Interaktionen und Handlungsabläufen, sondern vielmehr auch in dem Erfordernis, Situationen und daraus resultierende Handlungsaufforderungen explizit konzeptuell zu modellieren und auf dieser Basis Aufgaben zu erzeugen. Wie hiermit im Rahmen der Konzeption von Situationen und Aufgaben im Projekt TEMA umgegangen wird, wurde in diesem Beitrag exemplarisch am Beispiel der praktisch-technischen Kompetenz als Teilkompetenz des unmittelbar auf die zu pflegende Person bezogenen Kompetenzbereichs fachdidaktisch bzw. berufs- und wirtschaftspädagogisch für die Testentwicklung dargestellt und begründet. Vergleichbare Modellierungen sind für die diagnostischreflexive und für die interaktiv-kommunikative Teilkompetenz entwickelt worden. Inwieweit die technologische Umsetzung gewährleistet, dass die Stimuli - einschließlich der schwierigkeitsbestimmenden Merkmale von den Probanden im intendierten Sinne wahrgenommen werden, ist Gegenstand weiterer Modellierungs- und Prüfschritte, auf die im Rahmen dieses Beitrags nicht genauer eingegangen werden konnte. Aus ethischen Gründen nur bedingt empirisch prüfbar ist demgegenüber - jenseits der im Projekt bereits eingeholten Experteneinschätzungen - zu welchem Grad die technologische Simulation realen patientenbezogenen Handlungsanforderungen entspricht.

Inwieweit die dargestellten theoretischen Konzeptualisierungen empirisch tragen, d.h. unter anderem, inwieweit es gelingt, die Teilkompetenzen über die vorgenommene Aufgabenmodellierung kohärent abzubilden, wird in einem Pretest der nächste Prüfschritt der im Projekt TEMA 
intendierten empirischen Kompetenzmodellierung berufsfachlicher Handlungskompetenz in der Pflege älterer Menschen sein.

\section{Literatur}

Achtenhagen, F./Winther, E. (2009). Konstruktvalidität von Simulationsaufgaben: Computergestützte Messung berufsfachlicher Kompetenz - am Beispiel der Ausbildung von Industriekaufleuten. Bericht an das Bundesministerium für Bildung und Forschung. Göttingen. http://www.bmbf.de/pubRD/Endbericht BMBF09.pdf (30-10-2013)

Ashworth, P./Bjorn, A./Déchanoz, G./Delmotte, N./Farmer, E./Bulanda Kordas, A./Kritstiansen, E./Kyriakidou, H./Slajmer-Japelj, M./Sorvettula, M./Stankova, M. (1987): People's needs for nurisng care. A European Study: a Study of nursing care needs and of the planning, implementation and evaluation of care provided by nurses in two selected groups of people in the European Region. Copenhagen: WHO. Regional Office for Europe.

BMBF (2011): Bekanntmachung des BMBF von Richtlinien zur Förderung von Forschung und Entwicklung auf dem Gebiet technologieorientierter Kompetenzmessung in der Berufsbildung im Bereich „Entwicklung und Erprobung von technologieorientierten Messinstrumenten zur Feststellung der beruflichen Handlungskompetenz am Ende der Ausbildung in ausgewählten Berufen auf nationaler Ebene" vom 15.2.2011. BMBF. Bonn. Download unter http://www.bmbf.de/foerderungen/15827.php (18-10-2012).

BMG (2009): Bericht des Beirats zur Überprüfung des Pflegebedürftigkeitsbegriffes. Berlin: Referat für Öffentlichkeitsarbeit. https://www. Bundesgesundheits ministerium.de/uploads/publications/Neuer-Pflegebeduertigkeitsbegr.pdf (08-112013).

Döring, O./Wittmann, E./Hartig, J./Weyland, U./Nauerth, A. (2011): Verbundprojekt: Entwicklung und Erprobung von technologieorientierten Messinstrumenten zur Feststellung der beruflichen Handlungskompetenz in der Pflege älterer Menschen (TEMA). Unveröffentlichter Zwischenbericht für die erste Projektphase. Berichtszeitraum: 12/2011 - 10/2012. Nürnberg: f-bb.

Fichtmüller, F./Walter, A. (2007): Pflege lernen. Empirische Begriffs- und Theoriebildung zum Wirkgefüge von Lernen und Lehren beruflichen Pflegehandelns, Göttingen: $\mathrm{V} \& \mathrm{R}$.

Friese, M. (2010): Didaktisch-curriculare Aspekte für Fachrichtungen und Fachrichtungsbereiche personenbezogener Dienstleistungsberufe. In: Pahl., P./Herkner, V. (Hg.) Handbuch Berufliche Fachrichtungen. Bielefeld: Bertelsmann, S. 311-327.

Geißel, B./Hedrich, M. (2011): Identifizierung von Barrieren der Störungsdiagnose in simulierten und realen Anforderungssituationen bei Elektronikern. In: Faßhauer, U./Aff, J./Fürstenau, B./Wuttke, E. (Hrsg.): Lehr-Lernforschung und Professionalisierung. Perspektiven der Berufsbildungsforschung. Opladen, Farmington Hills, Mich.: Budrich 2011, S. 11-23. 
Gschwendtner, T./Geißel, B./Nickolaus, R. (2010): Modellierung beruflicher Fachkompetenz in der gewerblich-technischen Grundbildung. Projekt Berufspädagogik. In: Klieme, E./Leutner, D./Kenk, M. (Hrsg.): Kompetenzmodellierung. Zwischenbilanz des DFG-Schwerpunktprogramms und Perspektiven des Forschungsansatzes. Beiheft 56 z. Zeitschrift für Pädagogik. Weinheim, Basel: Beltz, S. 258269.

Haertel, E. H./Wiley, D. E. (1993): Representations of ability structures. Implications for testing. In: Frederiksen, N./Mislevy, R. J./Bejar, I.I. (Eds.). Test theory for a new generation of tests. Hillsdale, NJ: Lawrence Erlbaum.

Hundenborn, G. (2007): Fallorientierte Didaktik in der Pflege. Grundlagen und Beispiele für Ausbildung und Prüfung. München: Urban \& Fischer.

Hundenborn, G./Brühe, R. (2007): Curriculum für den Modellversuch „Erprobung einer Ausbildung in der Alten-, Kranken- und Kinderkrankenpflege mit generalistischer Ausbildung“. Düsseldorf: Ministerium für Arbeit, Gesundheit und Soziales des Landes Nordrhein-Westfalen. http://www.dip.de/file admin/ data/pdf/material/HundenbornBr\%C3\%BChe_2007Curriculum_generalistische_ Pflegeausbildung.pdf (13-11-2013).

Kaiser, F.-J. (1985): Fallstudie. In Lenzen, D. (Hrsg.): Enzyklopädie Erziehungswissenschaft. Bd. 4. Methoden und Medien der Erziehung und des Unterrichts. Stuttgart: Klett-Cotta, S. 440-444.

Klieme, E./Leutner, D. (2006): Kompetenzmodelle zur Erfassung individueller Lernergebnisse und zur Bilanzierung von Bildungsergebnissen. Beschreibung eines neu eingerichteten Schwerpunktprogramms der DFG. Zeitschrift für Pädagogik, 52. Bd., H. 6, S. 876-903.

Messick, S. (1994): The interplay of evidence and consequences in the validation of performance assessments. Educational Researcher, 23. Bd., H. 2, S. 13-23.

Mislevy, R. J./Steinberg, L. S./Almond, R. G. (1999): On the Roles of Task Model Variables in Assessment Design. CSE Technical Report 500. January 1999. CRESST/CSE. UCLA. Los Angeles. http://www.cse.ucla.edu/products/Reports/ TECH500.pdf (30-10-2013).

Mislevy, R. J./Almond, R. G./Lukas, J. F. (2004): A brief introduction to evidencecentered design. Princeton, NJ: ETS. http://marces.org/EDMS623/Mislevy\%20 on\%20ECD.pdf (30-10-2013).

Pätzold, G. (2006): Vermittlung von Fachkompetenz in der Berufsbildung. In: Arnold, R./Lipsmeier, A. (Hrsg.): Handbuch der Berufsbildung. Opladen: VS, S. 174190.

WHO (1996): Nursing Practice. Report of a WHO Expert Committee on Nursing Practice. Genf: WHO.

Wingenfeld, K./Büscher, A./Gansweid, B. (2008): Das neue Begutachtungsassessment zur Feststellung von Pflegebedürftigkeit. Projektbericht des Instituts für Pflegewissenschaft an der Universität Bielefeld und des Med. Dienstes der Krankenversicherung Westfalen-Lippe. http://www.uni-bielefeld.de/gesundhw/ ag6/downloads/Abschlussbericht_IPW_MDKWL_25.03.08.pdf (08-11-2011).

Wittmann, E. (2003): Zur Authentizität eines mündlichenen Prüfungsgesprächs im Ausbildungsbetrieb - Eine Untersuchung bei auszubildenden Bankkaufleuten. In: Buer, J. van/Zlatkin-Troitschanskaia, O. (Hrsg.): Berufliche Bildung auf dem Prüfstand. Entwicklung zwischen systematischer Steuerung, Transformation 
durch Modellversuche und unterrichtlicher Innovation. Frankfurt: Lang, S. 301325.

Wittmann, E./Krell, J./Simon, J./Worofka, I./Purwins, C. (in Vorbereitung): Herausfordernde Situationen in unterschiedlichen Settings der Pflege älterer Menschen. Otto-Friedrich-Universität Bamberg. 